\title{
The autophagy regulators Ambra1 and Beclin 1 are required for adult neurogenesis in the brain subventricular zone
}

\author{
M Yazdankhah ${ }^{1,2}$, S Farioli-Vecchioli ${ }^{3}$, AB Tonchev ${ }^{4}$, A Stoykova $^{4}$ and F Cecconi ${ }^{*, 1,2,5}$
}

Autophagy is a conserved proteolytic mechanism required for maintaining cellular homeostasis. The role of this process in vertebrate neural development is related to metabolic needs and stress responses, even though the importance of its progression has been observed in a number of circumstances, both in embryonic and in postnatal differentiating tissues. Here we show that the proautophagic proteins Ambra1 and Beclin 1, involved in the initial steps of autophagosome formation, are highly expressed in the adult subventricular zone (SVZ), whereas their downregulation in adult neural stem cells in vitro leads to a decrease in cell proliferation, an increase in basal apoptosis and an augmented sensitivity to DNA-damage-induced death. Further, Beclin 1 heterozygosis in vivo results in a significant reduction of proliferating cells and immature neurons in the SVZ, accompanied by a marked increase in apoptotic cell death. In sum, we propose that Ambra1- and Beclin 1-mediated autophagy plays a crucial role in adult neurogenesis, by controlling the survival of neural precursor cells.

Cell Death and Disease (2014) 5, e1403; doi:10.1038/cddis.2014.358; published online 4 September 2014

In the adult mammalian brain, neural stem cells are localized in two regions: in the subventricular zone (SVZ), a layer extending along the wall of the lateral ventricle, and in the subgranular zone of the dentate gyrus in the hippocampus. ${ }^{1}$ SVZ stem cells are strictly controlled under physiological conditions and are believed to replenish dying cells. In addition to their effect in maintaining brain homeostasis, they are also involved in neuronal replacement in response to injury. ${ }^{2}$ Although several factors are known to affect neurogenesis, understanding of the mechanisms that regulate adult neurogenic niches and their metabolism is still incomplete. Macroautophagy (hereafter referred to as autophagy) is an evolutionarily conserved cellular turnover process in which bulk cytoplasmic materials, long-lived proteins or damaged organelles are sequestered and delivered to lysosomes for degradation. $^{3}$ A complex crosstalk takes place between apoptosis and autophagy that determines the death or life of cells. ${ }^{4}$ Beclin 1 has a key role in autophagy initiation; ${ }^{5}$ it regulates the autophagy-promoting activity of the Class III $\mathrm{PI}$ 3-kinase Vps34, ${ }^{6}$ and is involved in the recruitment of membranes to form the key autophagy vesicles, named autophagosomes. Beclin 1 also interacts with $\mathrm{Bcl}-2,{ }^{7}$ and plays an important function in the regulation of cell survival. ${ }^{8}$ Ambra1 (activating molecule in Beclin 1-regulated autophagy) is another modulator of autophagy, which is phosphorylated by the upstream autophagy kinase Ulk1 and acts on Ulk1 stability and function. ${ }^{9,10}$ Ambra1 also interacts with Beclin 1 upon autophagic stimuli, thereby promoting the binding between Beclin 1 and its target kinase, Vps34. The binding between Ambra1 and mitochondrial Bcl-2 is also important for cell survival. ${ }^{11}$ Moreover, Ambra1 is crucial for nervous system development and is expressed from early neurulation onwards, with a high specificity for the neural plate. ${ }^{12}$

In contrast with studies on the pro-survival impact of autophagy in post-mitotic cells and in disease models, the role of autophagy in the maintenance and function of adult neural stem cells (ANSCs) is poorly understood. Here we have found that expression of upstream autophagy-regulating genes in the adult neurogenic region of SVZ, in physiological conditions, plays a crucial role in the regulation of adult neurogenesis.

\section{Results and Discussion \\ To dissect the autophagy molecular pathway involved in mammalian adult neurogenesis, we decided to analyze the expression levels of upstream proautophagic proteins in the SVZ of adult mouse brain, the niche of proliferating neural precursor cells, under physiological conditions. To this end, we first checked for the expression of Ambra1 and Beclin 1 (two members of the autophagy-initiation complex) in the SVZ. Ambra1 and Beclin 1 are expressed both in SVZ}

\footnotetext{
${ }^{1}$ IRCCS Fondazione Santa Lucia, Rome 00143, Italy; ${ }^{2}$ Department of Biology, University of Rome Tor Vergata, Rome 00133, Italy; ${ }^{3}$ Institute of Cell Biology and Neurobiology, National Research Council, Rome, Italy; ${ }^{4}$ Research Group in Molecular Developmental Neurobiology, Max-Planck Institute for Biophysical Chemistry, Göttingen 37077, Germany and ${ }^{5}$ Unit of Cell Stress and Survival, Danish Cancer Society Research Center, Copenhagen 2100, Denmark

${ }^{*}$ Corresponding author: F Cecconi, Department of Biology - University of Rome Tor Vergata, Via della Ricerca Scientifica, Rome 00133 , Italy. Tel: +39 06 72594230; Fax: +39 06 72594222; E-mails: francesco.cecconi@uniroma2.it or cecconi@cancer.dk

Abbreviations: Ambra1, activating molecule in Beclin 1-regulated autophagy; LC3, microtubule-associated protein 1 light chain 3; CHQ, chloroquine; Dapi, 4,6-diamidino-2-phenylindole; TuJ1, neuron-specific beta III tubulin; SVZ, subventricular zone; RMS, rostral migratory stream; OB, olfactory bulb; LV, lateral ventricle; ANSCs, adult neural stem cells; CCasp3, cleaved caspase-3; CPARP, cleaved poly (ADP-ribose) polymerase; DCX, doublecortin; 3-MA, 3-methyl-adenine; GFP, green fluorescent protein; MAP2, microtubule-associated protein 2; EGF, epidermal growth factor; FGF, fibroblast growth factor

Received 03.6.14; revised 17.7.14; accepted 21.7.14; Edited by A Verkhratsky
} 
nestin-positive neural stem cells (Figures $1 \mathrm{~A}$ and $\mathrm{C}$ ) and in doublecortin-positive neural progenitor cells (Figures 1B and D). Next, green fluorescent protein-microtubule-associated protein 1 light chain 3 (GFP-LC3) mice ${ }^{13}$ were used to check the levels of LC3 (a mammalian homolog of yeast Atg8, and one of the few reliable markers of autophagosomes) in the SVZ. This protein, normally diffused in the cytosol, decorates onforming autophagosomes once autophagy proceeds and its staining becomes punctate and easily detectable by immunofluorescence on ex vivo brain tissues. ${ }^{12}$ As expected, cells exhibiting a high expression of GFP-LC3 were largely present in the SVZ bordering the striatal wall of the lateral ventricles, a key area for adult neurogenesis (Figure 2a). By contrast, GFP-LC3-positive cells were virtually absent in the dorsal wall of the lateral ventricles, where neurogenesis is observed less frequently. As shown in Figure $2 \mathrm{~b}$, a strong GFP-LC3 signal is also detectable in migrating neuroblasts or immature neurons through the rostral migratory stream, visualized by neuron-specific beta III tubulin (TuJ1). ${ }^{14}$ High levels of GFP-LC3 signal were also detected in MAP2 (microtubule-associated protein 2)-positive mature neurons of the olfactory bulbs (Figure 2c). Taken together, these observations strongly suggest a role for Ambra1/Beclin 1-signaled autophagy in adult neurogenesis. It should be noted that, in accordance with these results, the mRNAs encoding Ambra1, Beclin 1 and endogenous LC3 show a very similar expression pattern in the adult mouse brain (as for the Allen Mouse Brain Atlas (Seattle, WA): Allen Institute for Brain Science).

Next, to evaluate the importance of autophagy in adult neurogenesis, ANSCs were isolated and expanded in culture from SVZs of 3-month-old wild-type, or Ambra $1^{+/ g t}$ and Beclin $1^{+/-}$mutant mice. Isolated ANSCs highly expressed the autophagy genes Ambra1, Beclin 1 and LC3 (Supplementary Figure S1A-C). To verify whether autophagy was also activated, the autophagic flux was blocked by chloroquine (CHQ) in ANSCs derived from autophagy-competent GFPLC3-transgenic mice. As a consequence, a bright GFP-LC3 signal appeared in proliferating (Ki67-positive) cells (Figure 3a). Blocking the autophagic flux with chloroquine led to the accumulation of autophagosomes, as shown by a further increase of LC3-II, detected by western blot analysis (Figure 3b). To confirm this finding, we also blocked autophagy by means of 3-methyl-adenine (3-MA), an inhibitor of class-III PI kinases (such as Vps34). This resulted in a significant reduction in neurosphere size from dissociated ANSCs, suggesting the existence of a role for autophagy during ANSC expansion (Supplementary Figure S2A). Similarly, a significant reduction of the autophagic flux could be observed in ANSCs derived from Beclin $1^{+/-}$mice, compared with wild-type littermates (Figure $3 \mathrm{~b}$ ). Thus, we decided to check whether the heterozygosity of Beclin 1 and autophagy impairment could affect ANSC proliferation. In line with our previous results, by counting the total number of cells after 7 days in culture, we observed a substantial decrease in the neurosphere-forming cell population derived from Beclin $1^{+/-}$mice, compared to wild-type cells (Figure 3c). Primary neurospheres were dissociated and seeded at low density, and we then measured the size of secondary spheres formed after 7 days of culture in vitro. We found that the size of secondary neurospheres from Beclin $1^{+/-}$mice was smaller and characterized by fewer Ki67-positive cells, compared to wild-type cells (Figure 3d and Supplementary Figure S2B). By contrast, the number of apoptotic cells in neurospheres derived from Beclin $1^{+/-}$mice increased significantly relative to the wild-type (Figure 3e). Taking together these results, we can hypothesize that basal autophagy is required for proper self-renewal and survival of ANSCs. Consistent with our findings, human hematopoietic stem cells fail to form colonies in colony-forming assays when autophagy is inhibited by siRNA against ATG5, another important autophagy progression gene. ${ }^{15}$ FIP200-mediated autophagy is also critical for maintenance of different types of stem cells by controlling the level of mitochondrial mass. ${ }^{16}$ Furthermore, more recent evidence demonstrates that autophagy is required for reprogramming of somatic cells to form induced pluripotent stem cells, and that Sox2-dependent temporal regulation of autophagy is a key step in cellular reprogramming processes. ${ }^{17}$ Sox2 is an essential transcription factor for maintaining self-renewal of stem cells. Thus, overall, autophagy could be important in the survival of ANSCs, controlling ANSCs' 'stemness' and sustaining the pool of stem cells in the SVZ niche.

Mechanisms of cellular homeostasis are important for preventing cellular injuries that could lead to impaired cellular function and ultimately to cell death: one of those mechanisms is autophagy. The autophagic activity within ANSCs may ensure their survival under conditions of tissue damage and organ dysfunction, thus allowing for subsequent repair. To verify this hypothesis, ANSCs were exposed to the genotoxic agent etoposide; cell resistance to death was then monitored. Under conditions of autophagy inhibition with 3-MA, ANSCs underwent apoptotic cell death (Supplementary Figure S3A). These results were further confirmed when etoposide was added to Beclin $1^{+/-}$neurospheres, in which apoptotic cell death was increased significantly relative to wild type (Supplementary Figure S3B). By means of these experiments, we thus demonstrate a pro-survival role of autophagy in ANSCs, even when they are stressed by a cytotoxic drug.

Expanded ANSCs were next allowed to differentiate for $72 \mathrm{~h}$ in differentiation medium, and we then monitored their neuronal hallmarks. By immunoblotting and immunofluorescence, we observed a marked increase in the levels of the TuJ1 ${ }^{18}$ (Supplementary Figure S4A and B). As mentioned before, GFP-LC3 is highly detectable in migrating immature neurons in the rostral migratory stream in vivo. As we hypothesized that autophagy may be functional to the early phase of neuronal differentiation, chloroquine was added to the differentiated GFP-LC3 ANSCs at different time points to block the autophagic flux and evaluate whether autophagosomes were accumulating or not. During the initial hours of differentiation of TuJ1-positive cells few GFP-LC3 dots were detected, but as differentiation proceeded the number of GFP-LC3 dots increased progressively (Supplementary Figure S4C). Taken together, our data argue for basal autophagy increasing in concomitance with the early steps of neuronal differentiation. A similar function has been previously proposed in human keratinocytes, in which autophagy constitutes an early signaling process required for keratinocyte commitment to differentiation. ${ }^{19}$ It seems that 
A

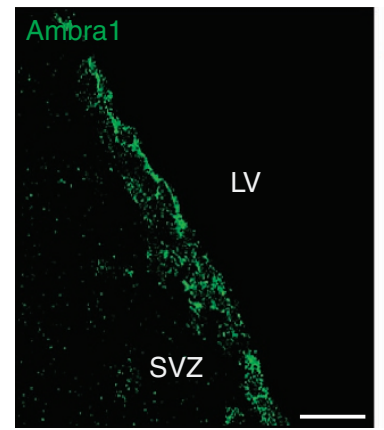

B a

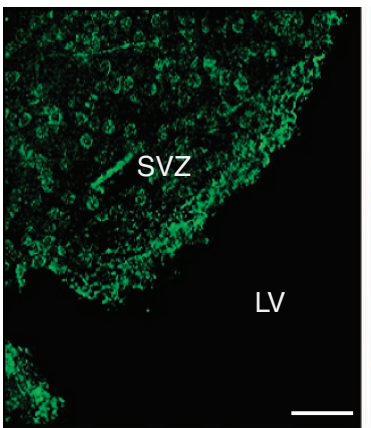

b

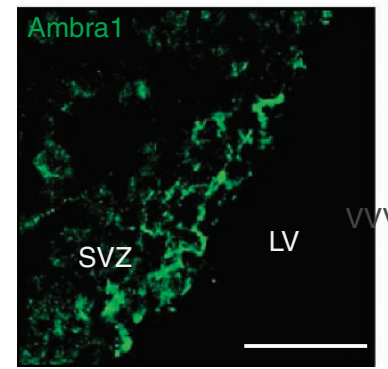

C

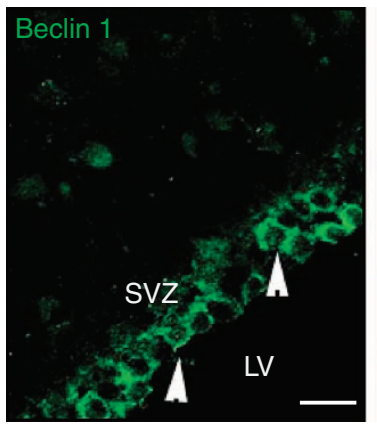

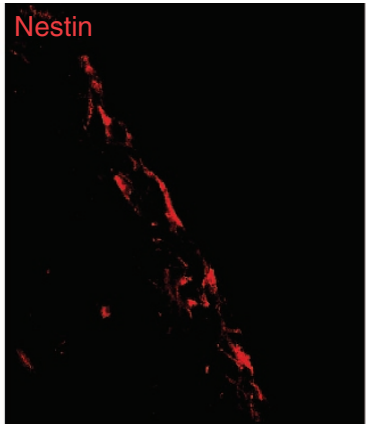
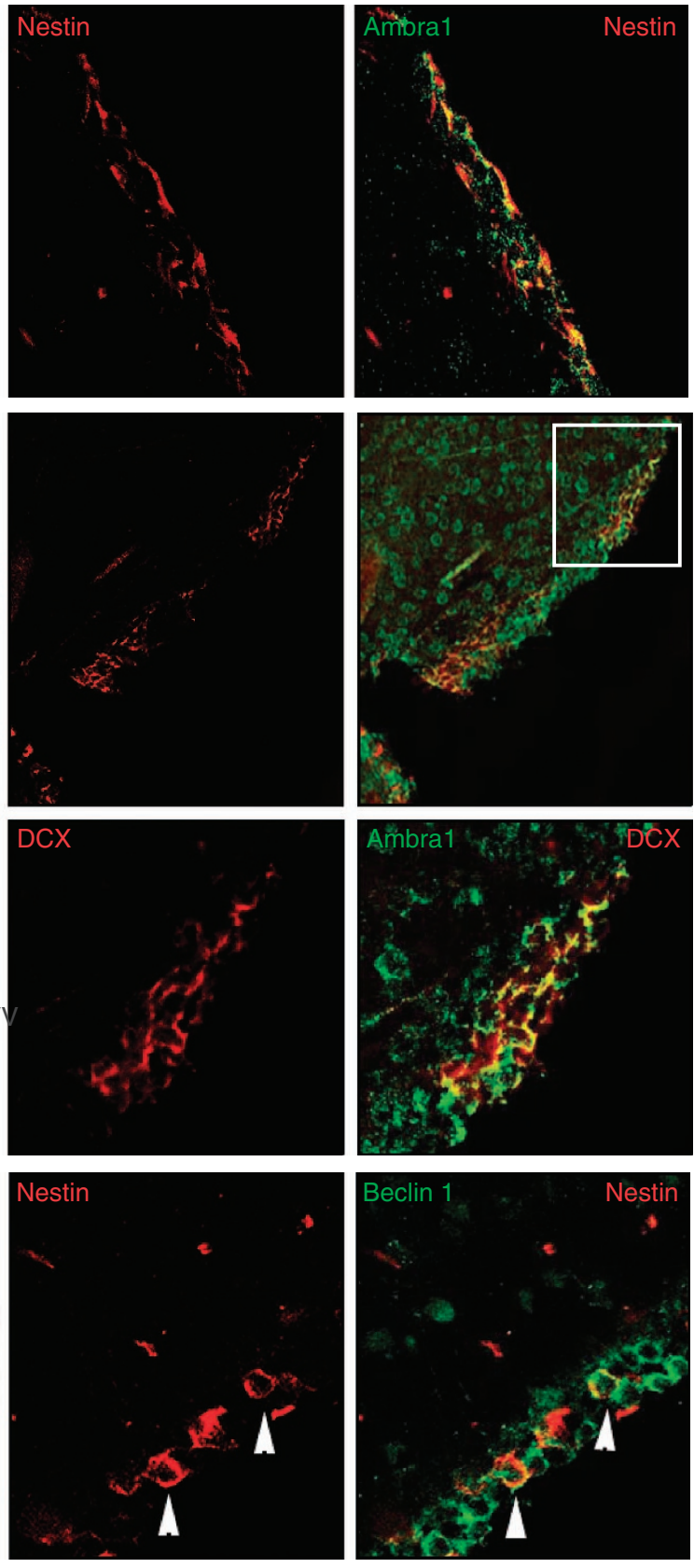

D
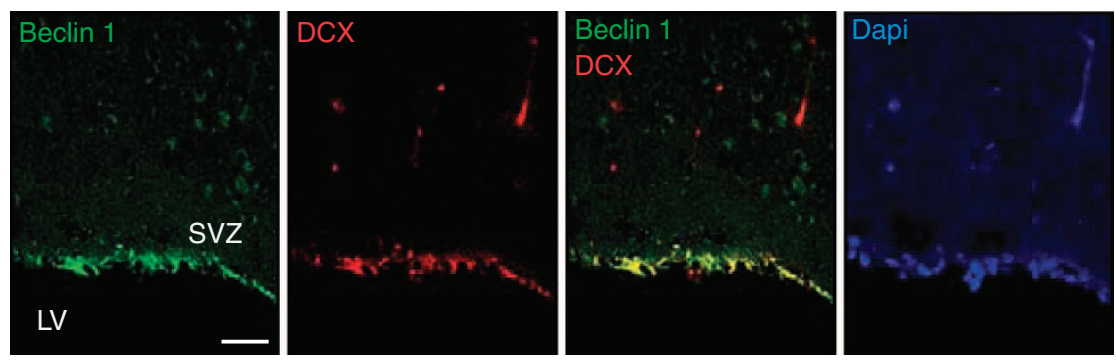

Figure 1 Expression of the proautophagic proteins, Ambra1 and Beclin 1, in the adult SVZ of the mouse brain. Immunohistofluorescent staining of adult brain coronal sections reveals Ambra1 and Beclin 1 expression in murine SVZ, under physiological conditions. Left panels, Ambra1 and Beclin 1 (green); middle panels, nestin and DCX (red); right panels, merged images. (A) Ambra1 is widely expressed in nestin-positive cells (scale bar, $20 \mu \mathrm{m}$ ). (B) Panel a: expression of Ambra1 in DCX-positive cells; Panel $\mathrm{b}$ : higher magnification $(\times 5)$ of the boxed region from Panel a (scale bar, $50 \mu \mathrm{m}$ ). (C) Nestin is expressed in Beclin 1-positive cells (arrowheads). (D) Co-expression of Beclin 1 and DCX in SVZ (scale bar, $20 \mu \mathrm{m}$ ). Dapi staining (blue) highlights the nucleus 


\section{a}
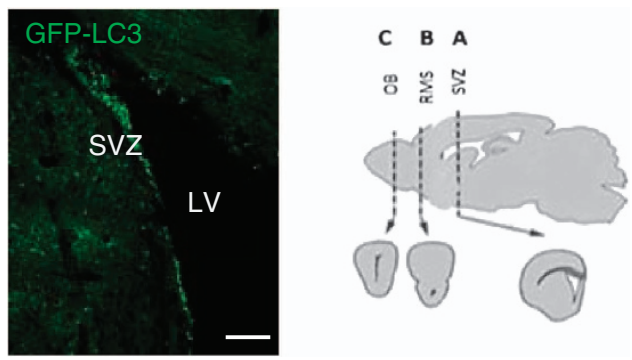

b
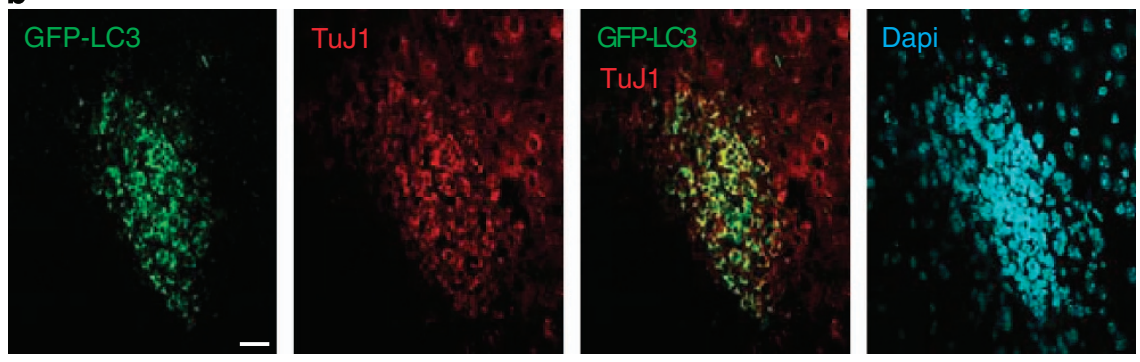

c
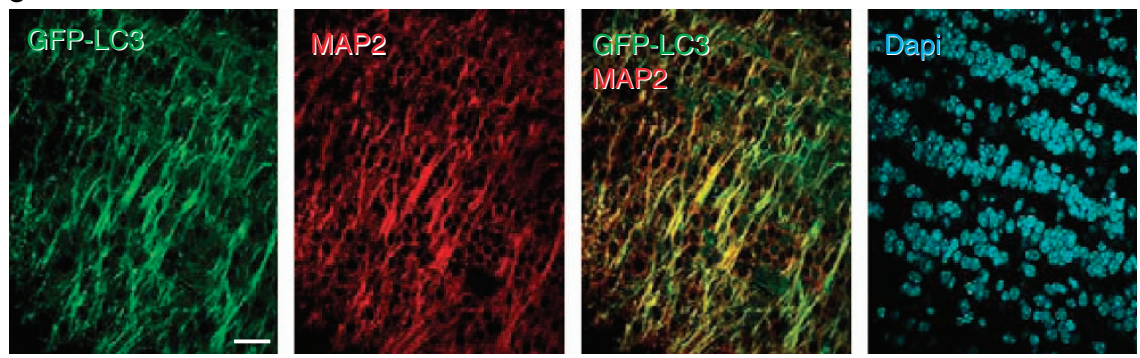

Figure 2 GFP-LC3 staining tags adult SVZ, RMS and OB. Representative images of coronal brain sections from GFP-LC3 transgenic mice (3 months old) taken through SVZ (A), RMS (B) and OB (C), as indicated in the diagram in (a). GFP-LC3 (green), anti-TuJ1 or anti-MAP2 (red), and Dapi (blue) staining was detected by a confocal microscope. (a) GFP-LC3 is expressed in the SVZ bordering the striatal wall of the lateral ventricles, a critical zone for adult neurogenesis. Note that, by contrast, GFP-LC3positive cells are virtually absent in the dorsal wall of the lateral ventricles, where neurogenesis is observed less frequently. (b) Also, the GFP-LC3 signal is widely detectable in migrating TuJ1-positive immature neurons along the RMS (scale bar, $20 \mu \mathrm{m}$ ). (c) Expression of GFP-LC3 in MAP2-positive mature neurons of the OB (scale bar, $20 \mu \mathrm{m}$ ). MAP2, microtubule-associated protein-2; TuJ1, neuron-specific beta-III tubulin

the impact of autophagy in differentiation is probably specific for different cell type populations. For instance, dermal, hematopoietic and epidermal stem cells show a decline in autophagy-related proteins during differentiation; ${ }^{20}$ on the other hand, autophagy activity seems to be upregulated during differentiation of cardiac stem cells and neural stem cells. $^{21-22}$ It should be noted that the autophagic flux was reduced when differentiation was induced in both $A m b r a 1^{+/ g t}$ and Beclin $1^{+/-}$ANSCs relative to wild type (Supplementary Figure S5A and B). Following reduction of the autophagic flux, the neuronal counts significantly declined (Figures $4 a$ and $c$ ) and, conversely, immunoblotting of apoptotic markers revealed an increase in cell death levels during differentiation of $A m b r a 1^{+/ g t}$ and Beclin $1^{+/-}$cells when compared with wild type (Figures $4 \mathrm{~b}$ and $\mathrm{d}$ ). These data demonstrate that reduction of neurogenesis due to autophagy impairment is a consequence of decreased survival of neural precursor cells during differentiation.

Based on these findings, we were further interested in evaluating whether an autophagy impairment could affect both proliferation and differentiation of precursor cells in the SVZ of 3-month-old Beclin $1^{+/-}$mice in vivo. The number of proliferating precursor cells was analyzed by the proliferation marker, Ki67: we observed a significant decrease in cell division when compared to wild type (Figure 5a). Conversely, the number of apoptotic cells (analyzed by cleaved-caspase 3 signal) in the SVZ of Beclin $1^{+/-}$mice increased significantly (Figure 5b). Moreover, there was a significant reduction in the number of TuJ1-positive cells in Beclin $1^{+/-}$mice relative to wild type, accompanied by increased cell death (Figures $5 c$ and $d$ ).

We can conclude that early autophagy regulators, like Ambra1 and Beclin 1, on the one hand sustain the stem cell pool within the adult SVZ and, on the other hand, control the level of immature neurons by enhancing the survival of neural precursor cells. Given the role of adult neurogenesis in response to brain injury and in some neurodegenerative diseases, the identification of key upstream genes and the elucidation of the impact of autophagy in this phenomenon may shed light on the 

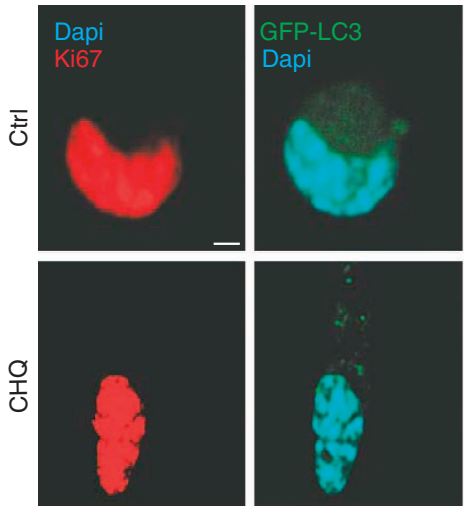

C

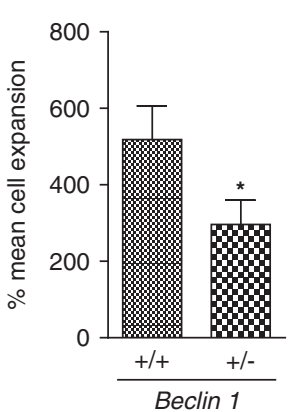

d

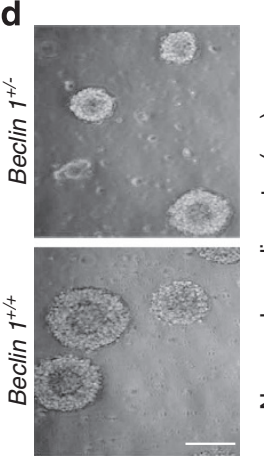

b
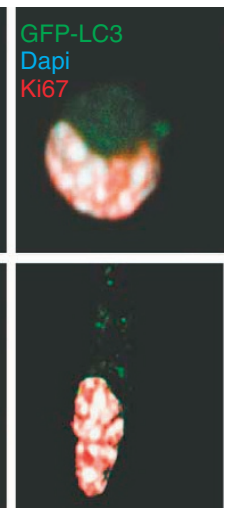
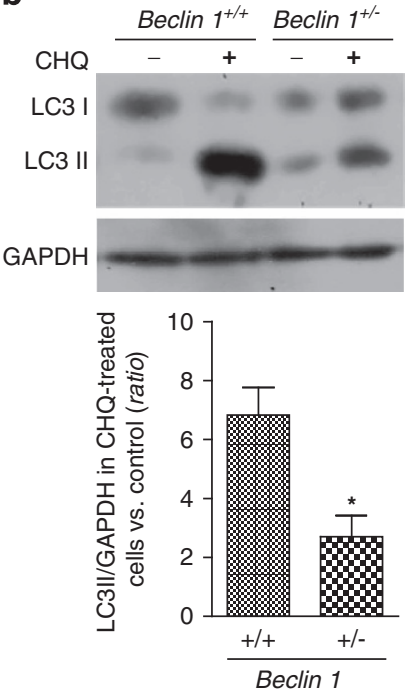

e
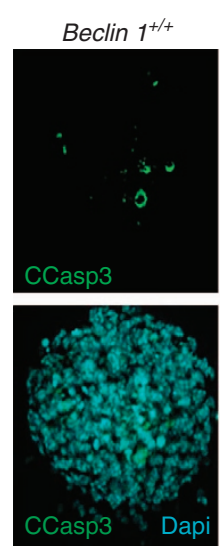

Beclin 1+-
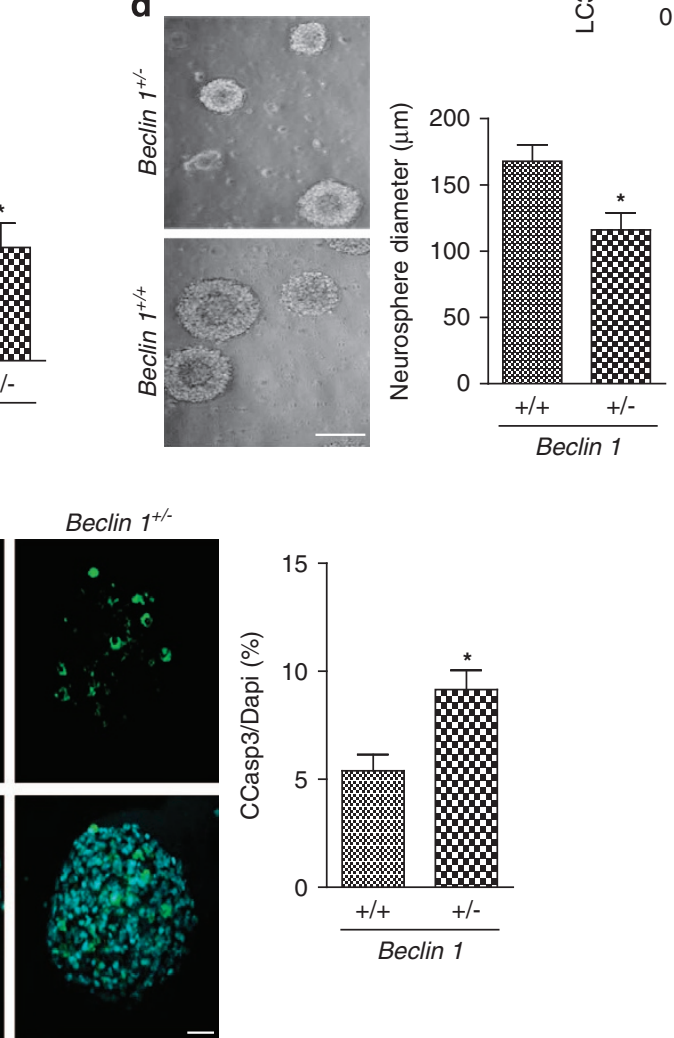

Figure 3 Autophagy occurrence inversely correlate with apoptotic cell death in aANSCs, grown in vitro. (a) Confocal microscope images of ANSCs in vitro, derived from GFP-LC3-transgenic mice. GFP-LC3 dots (green dots) and proliferating cells (Ki67, red) were detected together with the nuclei (stained by Dapi, blue). Blocking the autophagy off-rate with CHQ (lower panels) leads to the accumulation of autophagosomes; as a consequence, numerous bright GFP-LC3 dots appear in proliferating (Ki67-positive) cells (scale bar, $2 \mu \mathrm{m}$ ). (b) ANSCs were isolated and expanded in culture from wild-type (Beclin $1^{+/+}$) and Beclin 1 heterozygous (Beclin $1^{+/-}$) brains in the presence or absence of the lysosomal inhibitor $\mathrm{CHQ}$ during the last hour of culture. Then proteins were extracted and analyzed by immunoblot for LC3. A significant decrease in the accumulation of the autophagy-positive LC3-II isoform in Beclin $1^{+/}$relative to wild type after CHQ treatment. GAPDH, loading control. The ratio of LC3-II/GAPDH in treated cells with CHQ relative to untreated is shown as mean \pm S.E.M. $(n=4)$. (c) Percentage of cell expansion in ANSCs from Beclin $1^{+/-}$and wild-type controls (expressed as total number of cells at the end time-point of the culture divided by the initial number of cells; represented as mean percentage \pm S.E.M.). Relative to control, a greater clonal expansion takes place in cells from wild-type mice, when compared with cells derived from Beclin $1^{+/-}$mice. (d) Representative images of secondary neurospheres derived from Beclin $1^{+/}$and wild-type littermates (scale bar, $150 \mu \mathrm{m}$ ). Diameter (mean \pm S.E.M.) of secondary neurospheres derived from Beclin $1^{+/-}$and wild-type mice. With respect to wild-type neurospheres, the size of Beclin $1^{+/}$neurospheres is significantly smaller. (e) Representative images of secondary neurospheres stained with an anti-active cleaved caspase-3 antibody (a signature of caspase-dependent apoptosis), derived from Beclin $1^{+/}$and wild-type controls. Dapi (blue) stains the nuclei. In the graph (right) is shown the percentage of apoptotic cell death in secondary neurospheres, detected as cells positive for CCasp3 over the total cell number. Apoptotic cell death in Beclin $1^{+/-}$neurospheres is significantly higher than in wild-type cells (scale bar, $20 \mu \mathrm{m}$ ). Values represent mean \pm S.E.M. $(n=7),{ }^{*} P<0.05$ 
a
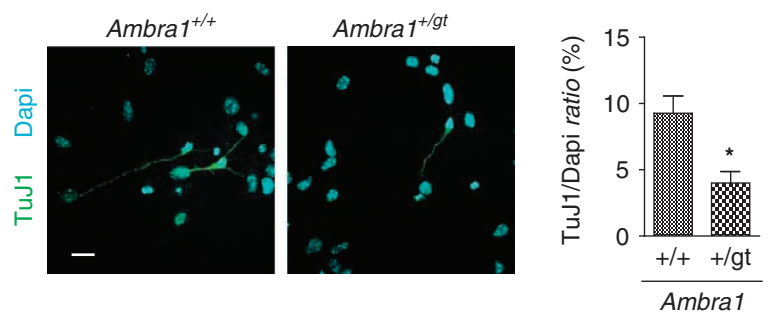

b
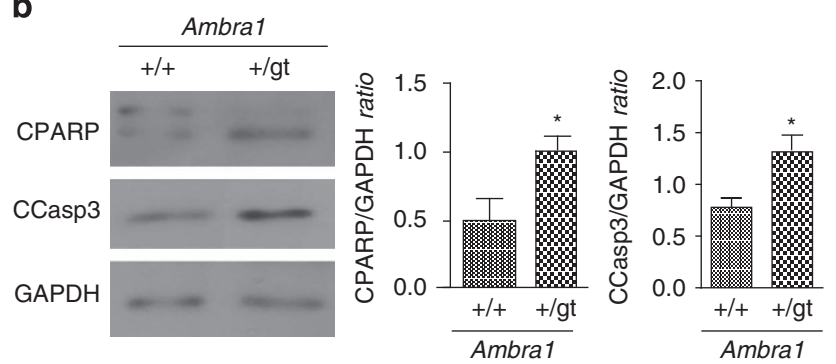

C
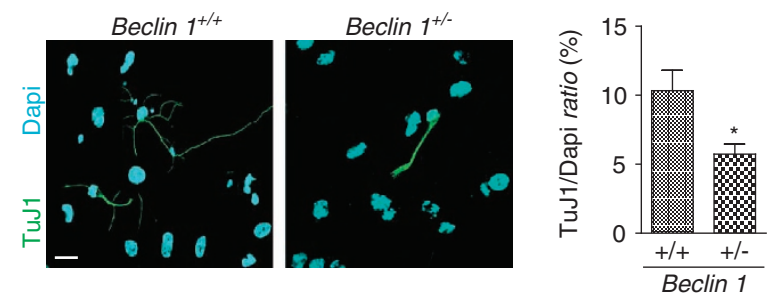

d
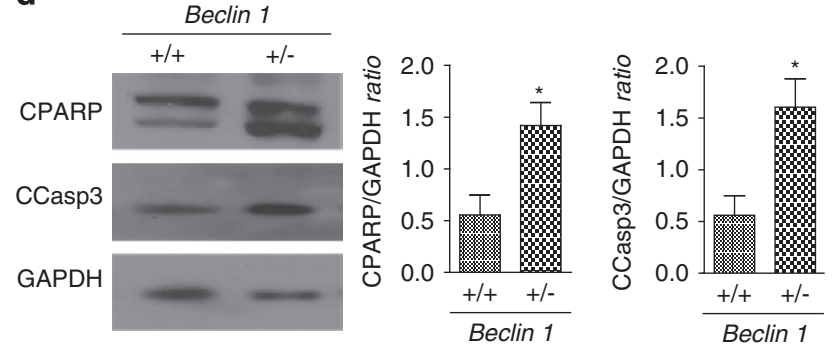

Figure 4 Impaired autophagy leads to decreased neuronal differentiation and increased apoptotic cell death in ANSCs in vitro. Ambra1 and Beclin 1 haploinsufficiency results in decreased neuronal differentiation. ANSCs from Ambra $1^{+/ g t}$ (heterozygotes), Beclin $1^{+/-}$and wild-type corresponding littermates were plated on matrigel-coated coverslips under differentiating conditions; after $72 \mathrm{~h}$ of culture, cells were fixed and stained for TuJ1 (green), and Dapi (blue). (a) Representative images of TuJ1-positive cells in differentiating Ambra $1^{+/ g t}$ and wild-type ANSCs. The percentage of TuJ1-positive cells (over the total Dapi-positive cells) is significantly reduced in Ambra1 ${ }^{+/ g t}$ relative to wild type (see also graph on the right). (b) Immunoblotting of ANSC protein extracts after $72 \mathrm{~h}$ of culture in differentiating condition. An increase in the expression of apoptotic markers (CCasp3 and CPARP) is clearly visible in Ambra $1^{+/ g t}$ cells relative to wild type. The graphs on the right summarize the densitometric analysis of the bands from six independent experiments repeated in triplicate. (c) Representative images of TuJ1-positive cells in differentiating Beclin $1^{+/-}$and wild-type ANSCs. The percentage of TuJ1-positive cells (over the total Dapi-positive cells) is significantly reduced in Beclin $1^{+/-}$relative to wild type (see also the graph on the right). (d) Immunoblotting of ANSC protein extracts after $72 \mathrm{~h}$ of culture in differentiating condition. An increase in the expression of apoptotic markers (CCasp3 and CPARP) is clearly visible also in Beclin $1^{+/-}$cells relative to wild type. The graphs on the right summarize the densitometric analysis of the bands from independent experiments repeated in triplicate. Values in all graphs represent mean \pm S.E.M., $(n=6),{ }^{*} P<0.05$. GAPDH, loading control molecular mechanisms involved in survival of precursor cells during regenerative processes.

\section{Materials and Methods}

Animals. Experiments were performed using adult C57BL6 mice (3 months old). GFP-LC3 mice were obtained from Dr N Mizushima and contain a transgene in which LC3 is fused to GFP and a CAG promoter. ${ }^{13}$ Transgenic Ambra1 mice were produced in our laboratory; they contain an insertion of the gene-trap vector within the Ambra1 gene. ${ }^{12}$ Heterozygous Beclin1 mice were obtained from $\operatorname{Dr} B$ Levine. ${ }^{23}$

Neural stem cell culture. Neural stem cell cultures were performed as described before. ${ }^{24}$ Neurospheres were grown in an incubator at $37^{\circ} \mathrm{C}$ in $5 \% \mathrm{CO}_{2}$ and cultured in DMEM/F12 medium supplemented with B27, epidermal growth factor and basic fibroblast growth factor ( 20 and $10 \mathrm{ng} / \mathrm{ml}$, respectively). Cells were passaged every 7 th day by mechanically dissociating neurospheres into single cells. To induce cell differentiation, cells from freshly dissociated neurospheres were seeded on matrigel (BD Biosciences, Bedford, MA, USA) coated cover-slips or dishes under differential conditions (complete medium without mitogens) for $72 \mathrm{~h}$. Differentiated cells were then fixed for immunostaining, or lysed for immunoblotting analysis. To analyze autophagic flux, chloroquine $(20 \mu \mathrm{M})$ was added to the culture medium to block the flux and accumulation of autophagosomes for $1 \mathrm{~h}$. To study autophagy inhibition 3-MA (10 mM) was added to the medium for $24 \mathrm{~h}$. Etoposide $(10 \mu \mathrm{M})$ was added to the medium for $24 \mathrm{~h}$.

Expansion capacity. Primary neurospheres were dissociated into single cells and plated at the same clonal density. Then, secondary neurospheres were dissociated and the number of cells was determined. The size of neurospheres was expressed as a volume calculated after measuring their diameter in phasecontrast pictures (assuming a spherical shape).

Immunofluorescence on neurospheres and microscopy. The differentiated cells on cover-slips were fixed with $4 \%$ paraformaldehyde in $0.1 \mathrm{M}$ phosphate buffered saline at room temperature for $30 \mathrm{~min}$. The cells were incubated for $60 \mathrm{~min}$ at room temperature with blocking solution containing $5 \%$ normal goat serum (Sigma-Aldrich, St. Louis, MO, USA) in $0.1 \mathrm{M}$ phosphate buffered saline containing $0.1 \%$ Triton $\mathrm{X}-100$. The blocking solution was replaced with fresh solution containing primary antibodies. The list of antibodies is reported in supplementary information. For CCasp3 immunostaining, neurospheres were plated on matrigel-coated coverslips for 20 min and then fixed in $4 \%$ paraformaldehyde for $2 \mathrm{~h}$ at RT. After fixation, neurospheres were permeabilized in $0.1 \%$ Triton $X-100$ in phosphate buffered saline and then incubated with the antibody against CCasp3 (Cell Signaling, Danvers, MA, USA). Immunostained neurospheres were mounted in Aquapolymount and analyzed using confocal microscopy (Zeiss LSM 700, Jena, Germany). Z-stack images were captured at $1 \mathrm{~mm}$ intervals with a $\times 63$ objective and a pinhole of 1.0 Airy unit. The number of CCasp3-positive cells was counted as a percentage of Dapi (4,6diamidino-2-phenylindole)-positive nuclei in four non-adjacent Z-stack images per neurosphere.

Immunohistochemistry. Tissue preparation and immunohistochemistry analysis were carried out by standard methods as described. ${ }^{24}$ Images of the immunostained sections were obtained by Zeiss confocal microscope and analyzed by using Zen Light Edition 2009 software (ZEN 2009 (Zeiss Enhanced Navigation) the free of charge digital image processing software (http:// www.softpedia.com/get/Multimedia/Graphic/Graphic-Viewers/ZEN-2009-LightEdition.shtml)). The list of antibodies is reported in supplementary information.

Gel electrophoresis and western blotting. Gel electrophoresis and western blotting were performed as described previously. ${ }^{9}$ Primary antibodies were rabbit anti-poly (ADP-ribose) polymerase and cleaved caspase-3 (Cell Signaling Technology), mouse anti-TuJ1 (Promega, Madison, WI, USA), rabbit anti-LC3 (Cell Signaling Technology), mouse anti-GAPDH (Millipore, Billerica, MA, USA). The optical density of specific bands was measured by densitometry. Densitometry analysis was carried out using ImageJ free image processing software (http://rsb.info.nih.gov/ij/). Protein levels were normalized relative to those of GAPDH. 
a
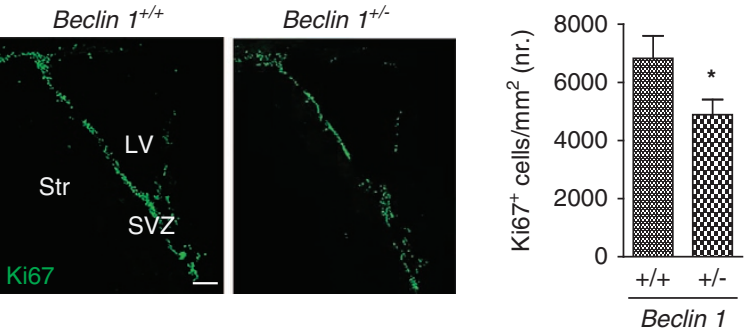

b
Beclin $1^{+/+}$
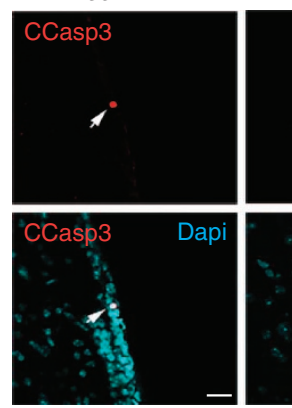

C
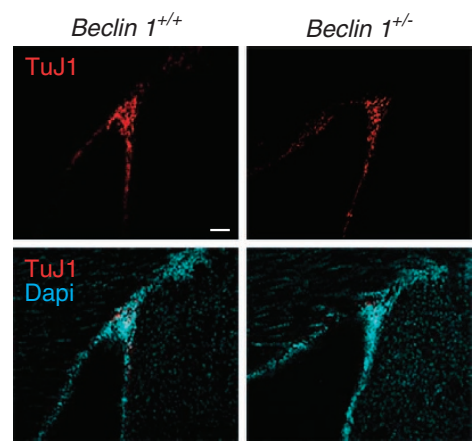

d
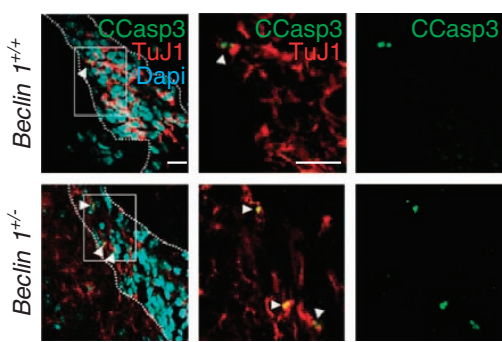

Beclin $1^{+/-}$
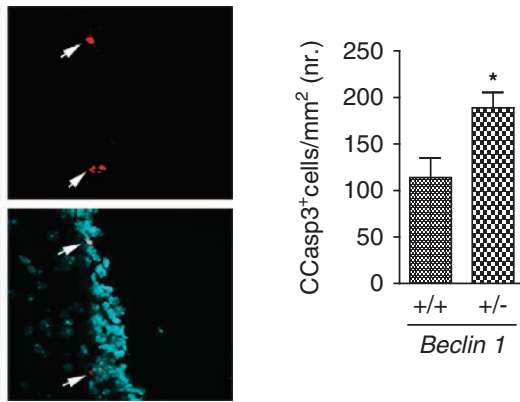

Statistical analysis. Results are expressed as the mean \pm s.e.m. of the number of independent experiments indicated in the figure legends. Statistical analyses were performed using Student's $t$-test. The level of significance was set at ${ }^{*} P<0.05 ;{ }^{*} P<0.01 ;{ }^{* *} P<0.001$.

\section{Conflict of Interest}

The authors declare no conflict of interest.

Acknowledgements. We are grateful to Raffaella Scardigli for her useful advices in the stem-cell culture set-up, to Martin W Bennett for manuscript editing and to Paola Merlo for critical discussion of this work. This work was supported in part by grants from the Telethon Foundation (GGP10225), AIRC (IG2010 and
Figure 5 Adult Beclin 1-deficient SVZs show decreased cell growth and increased apoptotic cell death. (a) Representative confocal microscope images of coronal sections showing proliferating (Ki67-positive) cells in the SVZ (scale bar, $100 \mu \mathrm{m}$ ). The graph (right) indicates that the number of proliferating cells per $\mathrm{mm}^{2}$ is significantly reduced in the SVZ of Beclin $1^{+/-}$mice relative to wild type. (b) Representative confocal microscope images of coronal sections showing apoptotic cells (detected as CCasp3-positive cells and indicated by white arrowheads) in the SVZ (scale bar $50 \mu \mathrm{m}$ ). The graph (right) indicates that the number of dying cells per $\mathrm{mm}^{2}$ is significantly increased in the SVZ of Beclin $1^{+/-}$ mice in comparison with the wild type. Dapi staining (blue) labels the cell nuclei. (c) Representative confocal microscope images of coronal sections showing TuJ1-positive immature neurons in the SVZ (scale bar, $50 \mu \mathrm{m}$ ). The graph (right) indicates that the number of immature neurons per $\mathrm{mm}^{2}$ is significantly decreased in the SVZ of Beclin $1^{+/-}$mice when compared with the wild type. (d) Representative confocal microscope images of apoptosizing (as revealed by CCasp3 staining, green and indicated by white arrowheads) TuJ1-positive (red) immature neurons (scale bar, $10 \mu \mathrm{m}$ ). The neurogenic area is outlined by a dashed line. Higher magnification of boxed regions corresponding to the insert is shown in the middle and right panels. The graph (right) indicates that cell death is increased in TuJ1-positive cells from Beclin $1^{+/-}$mice relative to wild type. Values represent mean \pm S.E.M. $(n=8) ;{ }^{*} P<0.05$

IG2013), FISM (2009), the Italian Ministry of University and Research (PRIN 2009 and FIRB Accordi di Programma 2011) and the Italian Ministry of Health (Ricerca Finalizzata and Ricerca Corrente to FC).

1. Limke TL, Rao MS. Neural stem cells in aging and disease. J Cell Mol Med 2002; 6: 475-496.

2. Deierborg T, Staflin K, Pesic J, Roybon L, Brundin P, Lundberg C. Absence of striatal newborn neurons with mature phenotype following defined striatal and cortical excitotoxic brain injuries. Exp Neurol 2009; 219: 363-367.

3. Cecconi F, Levine B. The role of autophagy in mammalian development: cell makeover rather than cell death. Dev Cell 2008; 15: 344-357.

4. Fimia GM, Corazzari M, Antonioli M, Piacentini M. Ambra1 at the crossroad between autophagy and cell death. Oncogene 2013; 32: 3311-3318.

5. Cao Y, Klionsky DJ. Physiological functions of Atg6/Beclin 1: a unique autophagy-related protein. Cell Res 2007; 17: 839-849.

6. Zeng $\mathrm{X}$, Overmeyer JH, Maltese WA. Functional specificity of the mammalian BeclinVps34 PI 3-kinase complex in macroautophagy versus endocytosis and lysosomal enzyme trafficking. J Cell Sci 2006; 119(Pt 2): 259-270.

7. Liang XH, Kleeman LK, Jiang HH, Gordon G, Goldman JE, Berry G et al. Protection against fatal Sindbis virus encephalitis by beclin, a novel Bcl-2-interacting protein. $J$ Virol 1998; 72: 8586-8596.

8. Chu CT, Zhu J, Dagda R. Beclin 1-independent pathway of damage-induced mitophagy and autophagic stress: implications for neurodegeneration and cell death. Autophagy 2007; 3: 663-666.

9. Nazio F, Strappazzon F, Antonioli M, Bielli P, Cianfanelli V, Bordi M et al. mTOR inhibits autophagy by controlling ULK1 ubiquitylation, self-association and function through AMBRA1 and TRAF6. Nat Cell Biol 2013; 15: 406-416.

10. Di Bartolomeo S, Corazzari M, Nazio F, Oliverio S, Lisi G, Antonioli M et al. The dynamic interaction of AMBRA1 with the dynein motor complex regulates mammalian autophagy. J Cell Biol 2010; 191: 155-168.

11. Strappazzon F, Vietri-Rudan M, Campello S, Nazio F, Florenzano F, Fimia GM et al. Mitochondrial BCL-2 inhibits AMBRA1-induced autophagy. EMBO J 2011; 30: 1195-1208.

12. Fimia GM, Stoykova A, Romagnoli A, Giunta L, Di Bartolomeo S, Nardacci R et al. Ambra1 regulates autophagy and development of the nervous system. Nature 2007; 447: 1121-1125.

13. Mizushima N. Methods for monitoring autophagy. Int J Biochem Cell Biol 2004; 36 : 2491-2502.

14. van Strien ME, van den Berge SA, Hol EM. Migrating neuroblasts in the adult human brain: a stream reduced to a trickle. Cell Res 2011; 21: 1523-1525.

15. Salemi S, Yousefi S, Constantinescu MA, Fey MF, Simon HU. Autophagy is required for self-renewal and differentiation of adult human stem cells. Cell Res 2012; 22: 432-435.

16. Wang C, Liang CC, Bian ZC, Zhu Y, Guan JL. FIP200 is required for maintenance and differentiation of postnatal neural stem cells. Nat Neurosci 2013; 16: 532-542.

17. Wang S, Xia P, Ye B, Huang G, Liu J, Fan Z. Transient Activation of Autophagy via Sox2Mediated Suppression of mTOR is an Important Early Step in Reprogramming to Pluripotency. Cell Stem Cell 2013; 13: 617-625.

18. Behrends C, Sowa ME, Gygi SP, Harper JW. Network organization of the human autophagy system. Nature 2010; 466: 68-76.

19. Aymard E, Barruche V, Naves T, Bordes S, Closs B, Verdier M et al. Autophagy in human keratinocytes: an early step of the differentiation? Exp Dermatol 2011; 20: 263-268. 
20. Pan H, Cai N, Li M, Liu GH, Izpisua Belmonte JC. Autophagic control of cell 'stemness' EMBO Mol Med 2013; 5: 327-331.

21. Vazquez P, Arroba Al, Cecconi F, de la Rosa EJ, Boya P, de Pablo F. Atg5 and Ambra1 differentially modulate neurogenesis in neural stem cells. Autophagy 2012; 8: 187-199.

22. Zhang J, Liu J, Liu L, McKeehan WL, Wang F. The fibroblast growth factor signaling axis controls cardiac stem cell differentiation through regulating autophagy. Autophagy 2012; 8: 690-691.

23. Qu X, Yu J, Bhagat G, Furuya N, Hibshoosh H, Troxel A et al. Promotion of tumorigenesis by heterozygous disruption of the beclin 1 autophagy gene. J Clin Invest 2003; 112: 1809-1820.

24. Farioli-Vecchioli S, Micheli L, Saraulli D, Ceccarelli M, Cannas S, Scardigli R et al. Btg1 is Required to Maintain the Pool of Stem and Progenitor Cells of the Dentate Gyrus and Subventricular Zone. Front Neurosci 2012; 6: 124.
Cell Death and Disease is an open-access journal published by Nature Publishing Group. This work is licensed under a Creative Commons Attribution-NonCommercialNoDerivs 3.0 Unported License. The images or other third party material in this article are included in the article's Creative Commons license, unless indicated otherwise in the credit line; if the material is not included under the Creative Commons license, users will need to obtain permission from the license holder to reproduce the material. To view a copy of this license, visit http://creativecommons.org/licenses/ by-nc-nd/3.0/

Supplementary Information accompanies this paper on Cell Death and Disease website (http://www.nature.com/cddis) 